\title{
Les Machines électriques à vitesse variable (sUite)
}

\section{Chapitre IV}

\section{Les moteurs monophasés}

1. - Généralités. - Les moteurs monophasés comprennent les machines survantes:

1. Le moteur d'induction simple,

2. Le moteur série à collecteur,

3. Le moteur à répulsion,

4. Le moteur à répulsion compensé, ou moteur Latour.

D'autres moteurs ont été mis sur le marché, mass nous nous limiterons aux machines cr-dessus, qui sont de beaucoup les plus répandues.

Il faut remarquer que les réseaux monophasés, en général moins économiques que les réseaux triphasés, tant au point de vue de la production et de la distribution que de l'utilisation du courant, ne trouvent leur plem avantage que dans deux catégories de problèmes :

A) Traction. - La ligne de transport monophasée est beaucoup plus simple que la ligne triphasée, et l'on peut aborder des tensions de distribution beaucoup plus élevées qu'en continu, avec la facilité qui résulte de l'emploi des transformateurs statıques, pour abaisser la tension.

в) Distributions urbaines. - La canalisation est plus simple . on peut brancher le moteur sur une ligne d'éclairage.

Il en résulte que les moteurs monophasés trouvent leur applcation fréquente :

a) Comme moteurs de traction de toutes puissances utilisables en traction.

b) Comme moteurs dans les distributions d'énergie alimentées directement par le réseau de traction (petites et moyennes puissances).

c) Comme moteurs dans les distributions urbaines à deux fils (petites et moyennes puissances).

2. - Moteur d'induction. - A) Description (fig. 70). - Ce
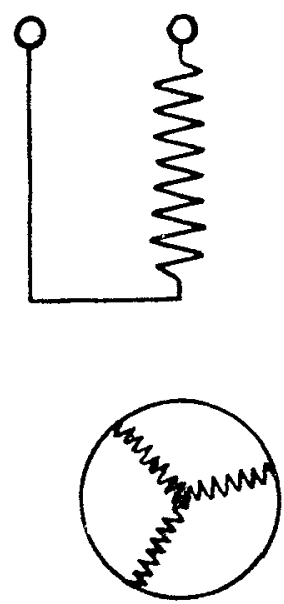

FIG. 70. - Moteur d'induction simple.

moteur se compose d'un stator monophasé alımenté par le réseau, et d'un rotor polyphasé, triphasé en général Un tel moteur doit être démarré à l'arde d'artıfices, car la simple fer- meture d'un interrupteur ne suffit pas. En général, on utilise un bobinage statorique auxiliaire, qui permet de démarrer en diphasé.

Caractéristiques. - $1^{\circ}$ Couple vitesse (fig. 71). — La caracté ristique est shunt, la vatesse à vide étant le synchronisme. It

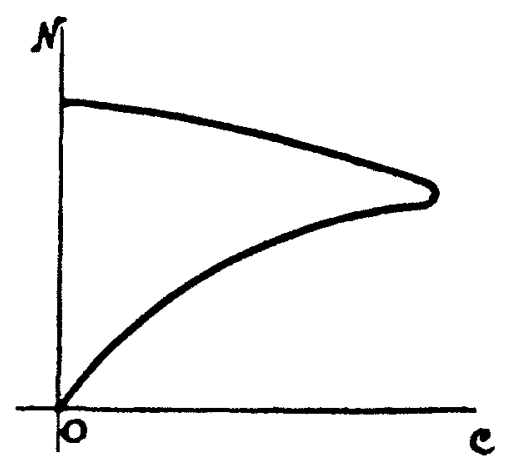

FIG. 71. - Moteur d'induction simple caractéristique couple-vitesse.

couple maximum est de l'ordre de 1,5 fois le couple normal (il est moins élevé que pour le moteur polyphasé).

La chute de vilesse entre marche à vide et marche en charg peut ètre réglée par insertion de résıstances dans le rotor, de li même façon que pour le moteur polyphasé. Mais le couple maxi: mum, au lieu de rester constant, diminue rapidement quand $k$ vitesse diminue. On est pratıquement limité à $20 \%$ de chut de vitesse.

$2^{\circ}$ Punssance déwattée. - Le moteur prend à vide la puis sance déwattée d'excitation. Le $\cos \varphi$ en charge est relativemelif faible, et ne dépasse pas la valeur 0,8 .

Couple au démarrage - Le couple moteur est nul au démar rage direct. Avec l'artifice du démarrage en diphasé, il est diffi cile d'obtenir, au démarrage, plus de $8 / 10$ du couple normi (et cela, avec un appel de courant qui attent plusieurs fois lt $^{2}$ courant normal).

En conclusion, ce moteur est loin de présenter des caractéri tiques aussi avantageuses que le moteur d'induction polyphase Aussi, est-il relativement peu répandu, et les moteurs monophas à collecteur ne rencontrent-ils pas en lui un concurrent sérieux.

3. - Moteur sérte compensé. - A) Description. - Ce mo teur est analogue au moteur série à courant continu : enroul ment d'excitation sérıe sur les pôles inducteurs, et rotor à collec teur avec deux lignes de balais à $180^{\circ}$ électriques.

Toutefois, il convient de lui adjoindre un bobinage de co pensation logé dans les pièces polaires de l'inducteur (dispos' tion analogue à celle qui est réalisée dans la machine à coural continu compensée). Le but de cet enroulement n'est pas tan d'annuler la distorsion (effet recherché en courant continu que de réduire le coefficient de self induction de l'induit en $\mathrm{w}$ d'obtenir un bon facteur de puissance.

Comme en courant continu, il convient d'utiliser des pâ de commutation placés suivant l'axe interpolaire. Ces pôl portent un enroulement série, qui est parfois shunté par résistance, en vue d'obtenir certames relations de phase favo bles à la commutation : on dit alors que le champ de commutati est déphasé. 
Le stator porte donc les bobinages d'excitation, de compensation, et de commutation (fig. 72), parfois plus ou moins combinés entre eux Dans la pratique, il est commode de réaliser la machıne avec un entrefer continu, les différents bobinages du stator étant logés dans des encoches de profondeurs mégales

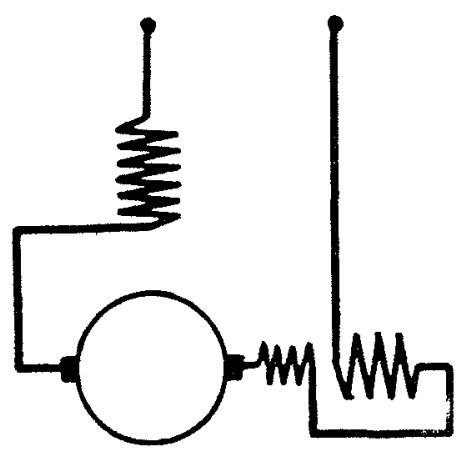

FIG. 72. - Moteur série compensé.

(dispositın qu est utilısée parfois avec les machınes à courant continu compensées) ; ll s'agit là, non d'une différence de principe par rapport à la machine à pôles salllants, mars d'un autre mode de réallsation

B) Caractéristzques. - a) Couple-vitesse - La caractéristique est série réglable par modification de la tension d'alimentation du moteur à chaque tension correspond une caractéristique différente. Pour une vitesse donnée, le couple varıe sensiblement, comme le carré de la tension il est maximum au démarrage

Les balais du moteur restent toujours fixes (dans cerlans moteurs, un léger réglage est prévu pour favoriser la commutation).
La vitesse de fonctionnement normale $N_{1}$ qui correspond aux meilleures conditions de commutation (vitesse au régime umborare en traction) est sensiblement supérieure à la vitesse de synchronisme : en général, 3 à 4 fois $\mathrm{Ns}$; cette condition nécessaire pour obtenrr un bon facteur de puissance.

b) Puissance déwattée (fıg. 73) - Pour une tension d'alimentation donnée, la pussance magnétisante prise au réseau

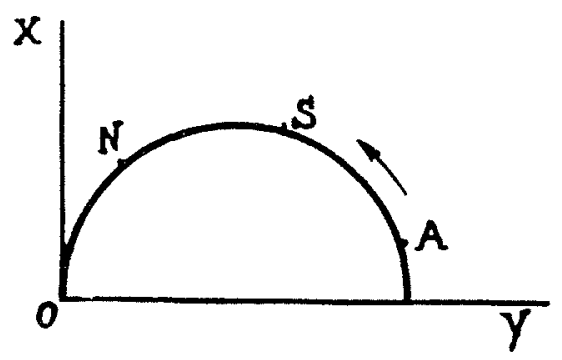

FIG 73. - Moteur série compensé : puissance réactıve.

est maxima à vide, et dıminue constamment quand la vitesse augmente.

A la vitesse normale, le $\cos \varphi$ est compris entre 0,85 (petits moteurs) et 0,90 (gros moteurs). Pour arriver à ces chiffres, il est nécessaire, comme nous l'avons déjà indıqué, que le moteur solt compensé, et tourne de 3 à 4 fois le synchronisme.

Le cos est d'autant plus élevé que la fréquence est plus faible.

La dragramme de courant est une circonférence passant par l'origine.

c) Vitesses et tensıons réalisables - Pour un couple donné, la vitesse peut être réglée dans de bonnes conditions dans le rap-

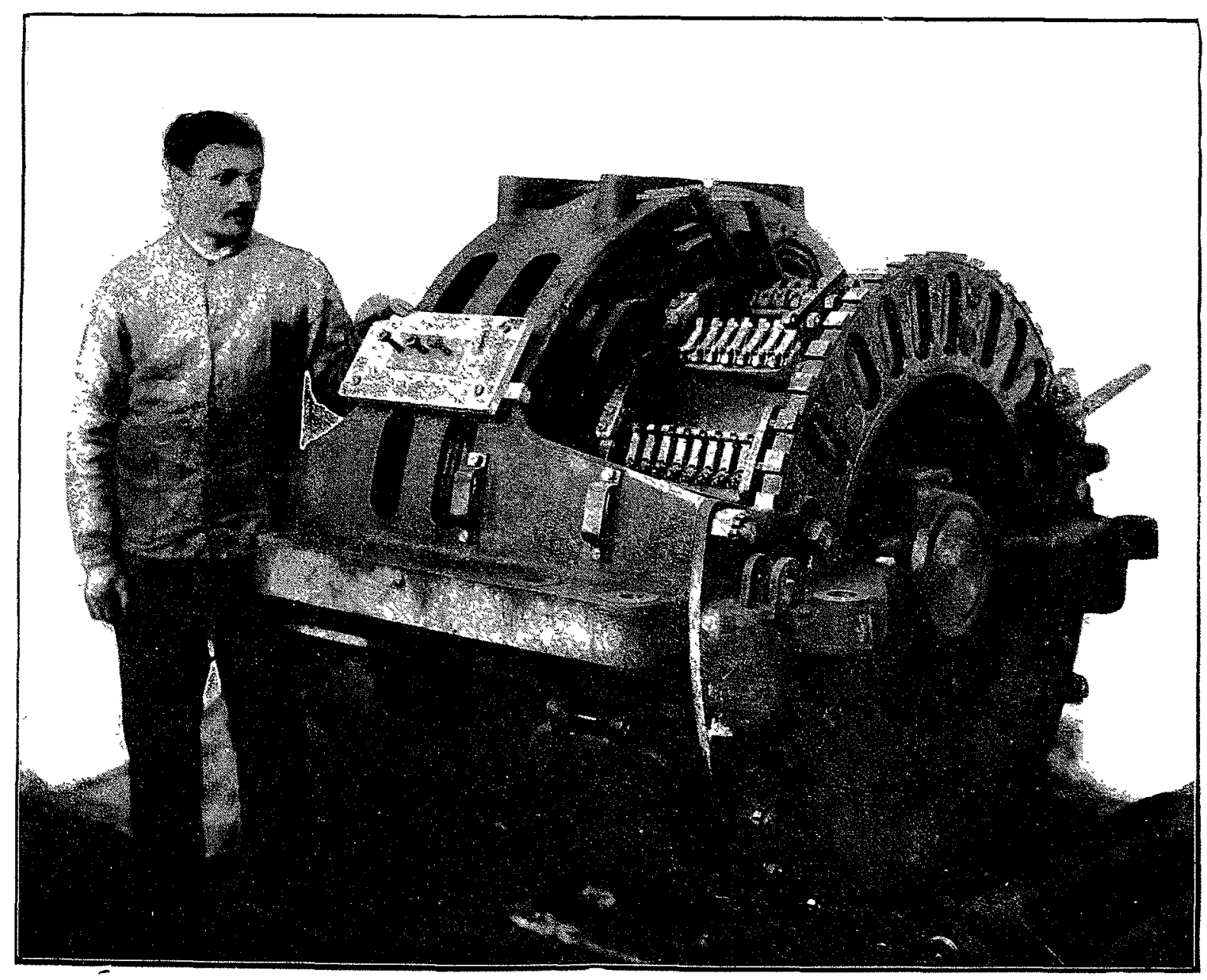

Moteur monophasé série compensé pour traction, $500 \mathrm{CV}$. (3 de ces moteurs ont été montés sur une locomotive électrıque de $1.500 \mathrm{CV}$. Compagnie des Chemuns de fer du Midi) 
port de 1 à 3 . Le moteu ne peut pas être ríalisé pour une lension quelconque - la tension qu convient dépend de la trequence d'alımentation el de la pussance.

D’une taçon générale, plus la tréquence est élevée, plus la tension dolt ètre basse $\$ 25$ pérıdes, 1 est diftucle de dépasser une tension de $150 \mathrm{~V}$. (petils moteurs) à $300 \mathrm{~V}$. (gros moteurs). A 16 périodes, ces lmmles s'élèvent à 250 et $500 \mathrm{~V}$.

Le moteur ne s'adaple donc pas à la tension d'un réseau, aussı faclement que le moteur à courant contmu ll est presque toujours associé à un transformateur, qu lur foum nit la tension la plus convenable, et, par sute, la lension de la hgne peut ètre chorsie aussi élevée qu'il convient pour le transport economique de l'energie.

La tension optma etant relativement basse, le collecteur el l'apparellage sont à prévour pour des coulants mporlants, ce qui est un inconvénent sémeux en traction

d) Couple au démarrage - Le moleur sére possède un couple élevé au démarrage, avec appel de courant rédul, le rapport de $\frac{\mathrm{C} d}{\mathrm{C} n}$ à $\frac{\mathrm{I} d}{\mathrm{I} n}$ est de l'oldre de 1,2 à $1, \overline{3}$.

e) Varıations de tension et de fréquence - Le moteur série est sensible aux variatıons de tension, comme indıqué plus haut.

Par contre, une variation de fréquence influe relatıvement peu; à la lımite, le moteur est susceptıble de fonctionner en courant continu (Certains moteurs de traction sont appelés à fonctionner successivement en courant contmu ou en monophasé, selon la partıe de ligne intéressée)

f) Couple négatıf. - Le fonctıonnement en couple négatıf sur le réseau présente certaines difficultés, car ll faut exciter le moteur par un courant de phase convenable : on utilise une excitatrice spéciale - tout comme dans le cas du moteur série à courant contınu - ce qu permet d'obtenir en couple négatif, une caractéristique shunt Certaines dispositions auxiliaires permettent d'obtenur un facteur de pussance élevé Le problème se pose principalement en traction, pour la récupération

4. - Moteur à répulsion. - A) Description. - Le moteur se compose d'un stator monophasé analogue à un stator d'alternateur, et d'un rotor à collecteur avec deux lignes de balais à $180^{\circ}$ électriques (fig. 74)

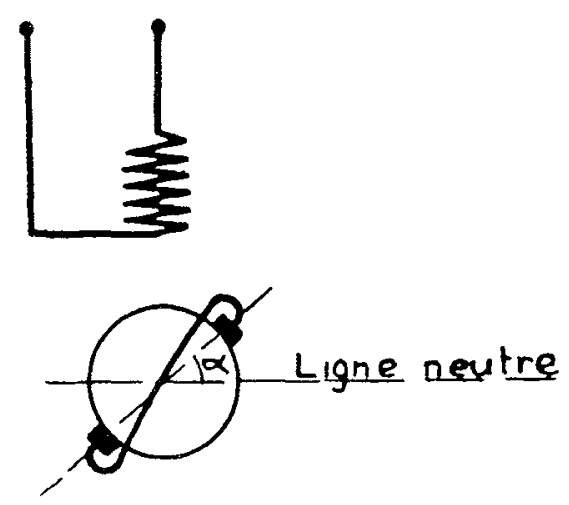

FIG. 74 - Moteur à répulsıon.

Le stator est relié au réseau, et les balais sont réunis par un court-circult.

Le moteur est réglé par décalage de l'ensemble des balais sur le collecteur. Dans la pratıque, on n'utılise pas deux lignes de balass par pare de pôles, mass qualre lignes formant deux courl-circuils dislancts. Cetle disposilion, due à M. Marıus Latour, permet d'améliorer la commutation en situant les balass dans une rógion où la lension entre lames est mondre. L'axe du bobmage rolorique est alors l'axe de symétrie ([1g. 75). Le moleur cst réglé par décalage de l'ensemble des balass.

Dans la position iniluale des balas (ligne neutre), l'axe du bobinage colorque esl normal à l'axe statorique Le inoleur
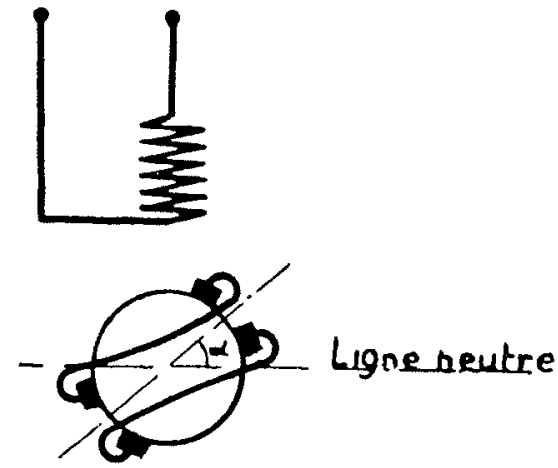

Fıg 75. - Moteur à répulsion avec court-circuits multıples.

démarre dans un sens ou dans l'autre, selon le sens du décalage. Celte propriélé de l'inversion du sens de rotation par simple décalage, est l'une des proprétés caracléristiques du molcur.

B) Caractéristiques. - a) Couple-vitesse - La caracléristıque est sérıe réglable par décalage des balaıs

La vitesse de fonctionnement normale, qu correspond aux melleures conditions de commutation, est égale à la vilesse de synchronisme $\mathrm{N}_{\mathrm{S}}$

A vide, le moteur s'emballe Il est possible de limiter la vitesse à vide en mettant le bobinage rolorıque en courl-circuit, à l'aude d'un conjoncteur centrifuge, ce qui transforme la machine en moteur d'induction monophasé. (Le court-circuil doıt être polyphasé : en général truphasé)

b) Puissance déwattée (fig 76). — A la ligne neutre, le moteur prend au réseau son courant magnétisant, qui est de l'ordre de

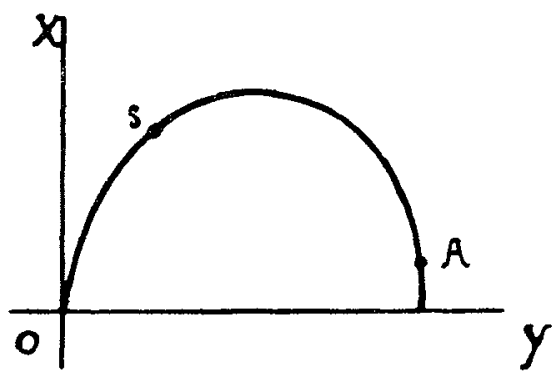

Fig. 76 - Moteur à répulsion, puissance réactıve pour un calage des balais.

30 à $50 \%$ du courant normal. En marche normale voisme du synchronisme, le $\cos \%$ attent la valeur 0,8 environ : $1 \mathrm{l}$ est toujours inférieur à celui du moteur série compensé. Le. $\cos \varphi$ est optrmum au vossinage du synchronisme, quand lc moteur est utrlisí à couple constant el vilesse variable

Le diagramme de courant esl une circonférence passant par l'origine.

Pour rédure la consommalion de puissance déwallée, dalls le cas d'un moteur fonctionnant à farble charge, on peut affarblir le champ (par modification du nombre des spires du stator), suivant divers artifices.

c) Couple au démarrage - A mesure que l'on décale les balais, à parır de la ligne neutre, le couple de démarrage aud 
mente, Il alleml un maxumum pour un calage un peu inférleur à $90^{\circ}$ ćlectruques, et s'annule pour celle dernère valeur (fig. 77).

Le rappoil $\frac{C_{d}}{C_{n}}$ à $\frac{I_{d}}{\Gamma_{n}}$ est de l'ordre de 1,2 à 1,3 .

La valeur maxmmum de $\frac{C_{d}}{C_{n}}$ peut atleindre 3 à 4 : ce couple élevé au démarrage est l'une des propriétés caractérıstiques du molem.

d) Vilesses el lensions réalisables. - La vitesse peut être réglée de $0,1 \mathrm{~N}$ à $1,1 \mathrm{~N}$ s dans de bonnes condilions · soll sen-

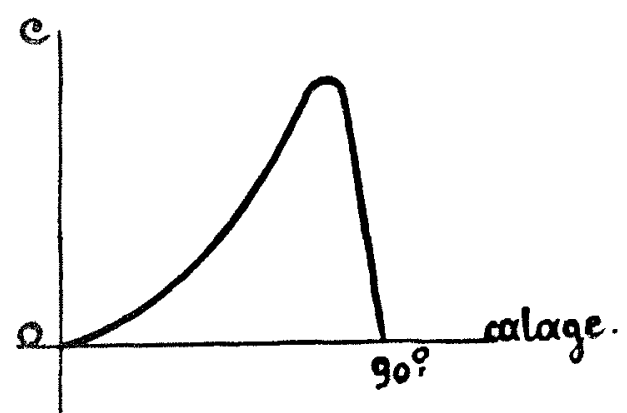

Fig. 77. - Moteur à répulsion, couple au démarrage en fonction du décalage.

siblenent de 1 à 3 . La question de commutation limite rapidement la variation réalisable au-dessus du synchronisme (1).

La tension d'alimentation peut être quelconque, car le collecteur est hors circuit . on peul réalıser les moteurs de moyenne et grande pussance à $6.000 \mathrm{~V}$, sans aucun transformateur.

Le moleur peut être construt, pour la fréquence 50 , sans diff-culté, tandıs que cetle fréquence étart trop élevée pour le moteur série.

Si l'on compare le moteur série compensé et le moteur à répulsion, à vitesses et puissances égales et fréquence égale du réseau, le moleur à répulsion aura un nombre de pôles 3 à 4 fors plus fable que celur du moteur série : la puissance par pôle du moteur à répulsıon est donc plus élevée.

D'autre part, au point de vue commutation, la commutation du moteur à répulsion est relativement moins bonne que celle dư moteur sérıe, quand on s'écarte de la vitesse optıma.

e) Couple négatıf. - Si on entraîne le moteur en sens inverse de celui qui est déterminé par le décalage des balais, ou sı, la machine tournant en moteur dans un sens déterminé, on décale les balais de l'autre côlé de la ligne neutre, la machine fonctionne en couple négatıf. Toutefors, ce fonctionnement est délicat, car la machine est susceptıble de s'amorcer à une fréquence propre inférleure à celle du réseau; il peut être nécessaire d'intercaler des résistances pour lımiter l'effet d'amorçage.

5. - Moteur Latour sérte. - A) Description. - Ce moteur se compose d'un rotor à collecteur, avec deux axes de balais par paire de pôles deux lignes diamétrales constituent l'axe du balais d'excilation et sont reliées en série avec le stator. Deux

(1) La rason pour laquelle la commutation est parfaite au synchronisme est la suivante : à cette vitesse, il existe deux flux clans la machune, dont les axes sont perpendicularres et dont les phases sont décalées de $90^{\circ}$. Ces deux flux donnent un champ tournant qui tourne à la même vitesse et dans le même sens que le rotor : aucune tension d'origine extérieure n'est donc induite dans les spires en commutation. La machine se comporte exactement comme un moteur biphasé à collecteur; elle est, à proprement parler, une machine à champ tournant, ce qui explique l'emploi d'un entrefer continu. lignes à 90 degrés de la précédente sont reliées par un courtcircuit, et constituent l'axe du court-circuit (fig. 78).

On réalıse parfois la disposition des courts-circuits multiples, comme dans le cas du moteur à répulsion.

La caractéristıque est réglable par décalage de l'ensemble des balais.

Dans le cas d'un réseau à tension élevée, il convient d'alımenter

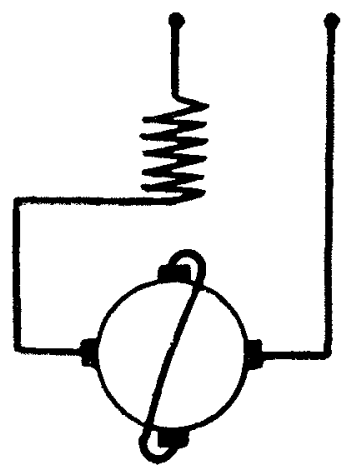

Fig. 78. - Moteur Latour série.

le rotor, non pas directement en sérle, mais par l'intermediaire d'un transformateur, dont la puissance est fauble par rapport à celle du moteur.

B) Caractérıstıques. - a) Couple-vitesse. - La caractérıstıque est série, réglable par décalage des balais.

Pour raisons de commutation, la vitesse de fonctionnement normale est le synchronisme

A vide, le moteur s'emballe; il est possıble de limiter la vitesse d'emballement à 1,5 fors la vitesse normale, par l'emplo1 d'une inductance connectée en dérivation aux balass.

b) Puissance déwattée (fıg 79) — La pussance déwattée à vide est comparable à celle du moteur à répulsion sımple.

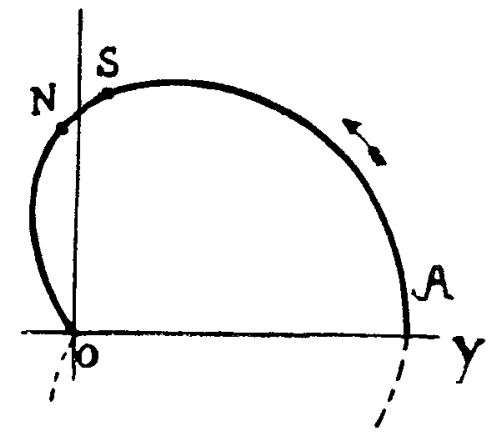

FIG. 79. - Moteur Latour série,

Puissance réactıve pour un calage des balais.

Le $\cos \phi$ en charge attent la valeur 1 au synchronisme, audessus du synchronisme, le moteur débite de la puissance réactive au réseau.

C'est là une propriété caractérıstıque du moteur Latour, quı constıtue sa principale supérıorité sur le moteur à répulsion.

Le diagramme de courant est une courbe fermée du quatrième degré qui a l'allure d'une cardionde.

c) Couple au démarrage. - Le couple au démarrage peut atteindre 3 à 4 fois le couple normal.

d) Vitesses et tensions réalisables. - La vitesse peut être réglée de 0,5 à $1,3 \mathrm{Ns}$ dans de bonnes conditıons.

Dans le cas d'un réseau à tension élevée, on utilısera. comme nous l'avons indıqué, un transformateur de rotor. 
Le moteur n'est pas indiqué en traction pour des puissances élevées, par suite de l'importance du collecteur sur lequel il faut placer deux jeux de balais différents; par contre, il est souvent employé en moyenne traction.

Le moteur peut être établi pour la fréquence 50.

e) Couple négatif. - Le moteur peut fonctionner en couple négatif sur le réseau pour un calage convenable des balais.

Il peut également fonctionner en autoexcitation sur résistances.

6. Moteur Latour série-shunt. - A) Description. - Grâce à l'emploi d'un enroulement auxıliaure, logé dans le stator, il est possible de convertır le moteur Latour série en moteur à caractéristique shunt, avec calage fixe des balais. On démarre en moteur série, et quand la vitesse de synchronisme est atteinte, on ferme l'enroulement shunt (automatiquement, à l'aide d'un conjoncteur centrifuge, par exemple) (fig. 80).

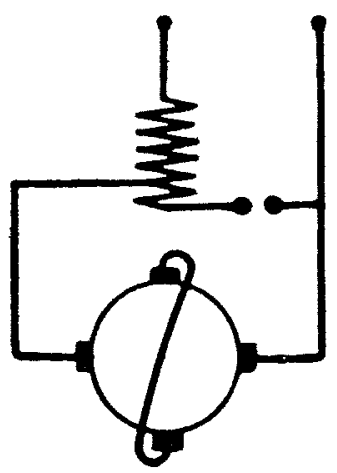

FIG. 80. - Moteur Latour série-shunt.

B) Caractéristiques. - a) Couple-vitesse. - Lorsque la connexion shunt est établie, le moteur fonctionne avec une caractéristique shunt : la vitesse à vide est le synchronısme et la vitesse en charge légèrement inférieure.
Le couple maximum est très élevé (plus élevé que pour moteur d'induction simple).

b) Puissance déwattée (fig. 81). - En connexions série, puissance déwattée prise au réseau, maxima au démarrage diminue à mesure que la vitesse augmente.

En connexions shunt, le facteur de puissance reste voisine

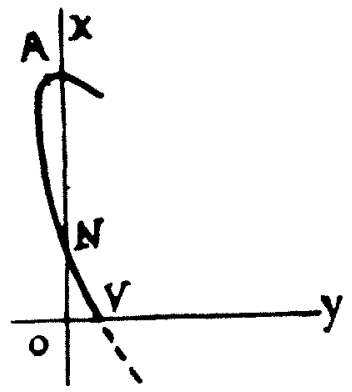

FIG. 81. - Moteur Latour shunt puissance réactive.

l'unité dans de grandes limites de variation de la charge; vide, les kVA pris sur le réseau sont faibles.

Le diagramme de courant est une courbe d'allure parabolique L'axe de la parabole peut être plus ou moins inclmé; ll pent arriver que le moteur débite à vide une puissance magnétisant sur le réseau.

c) Couple au démarrage. - On se reportera à ce qui a été dit pour le moteur Latour série

d) Vitesses et tensions réalisables. - La vitesse doit être un vitesse de synchronisme : le moteur peut être - dans tousle cas - employé au lieu du moteur d'induction, avec les avant tages quı luı sont propres. La commutation est parfaite à toule charge.

e) Couple négatif - Le moteur fonctionne en couple négati sur le réseau, à une vitesse légèrement hypersynchrone, ave

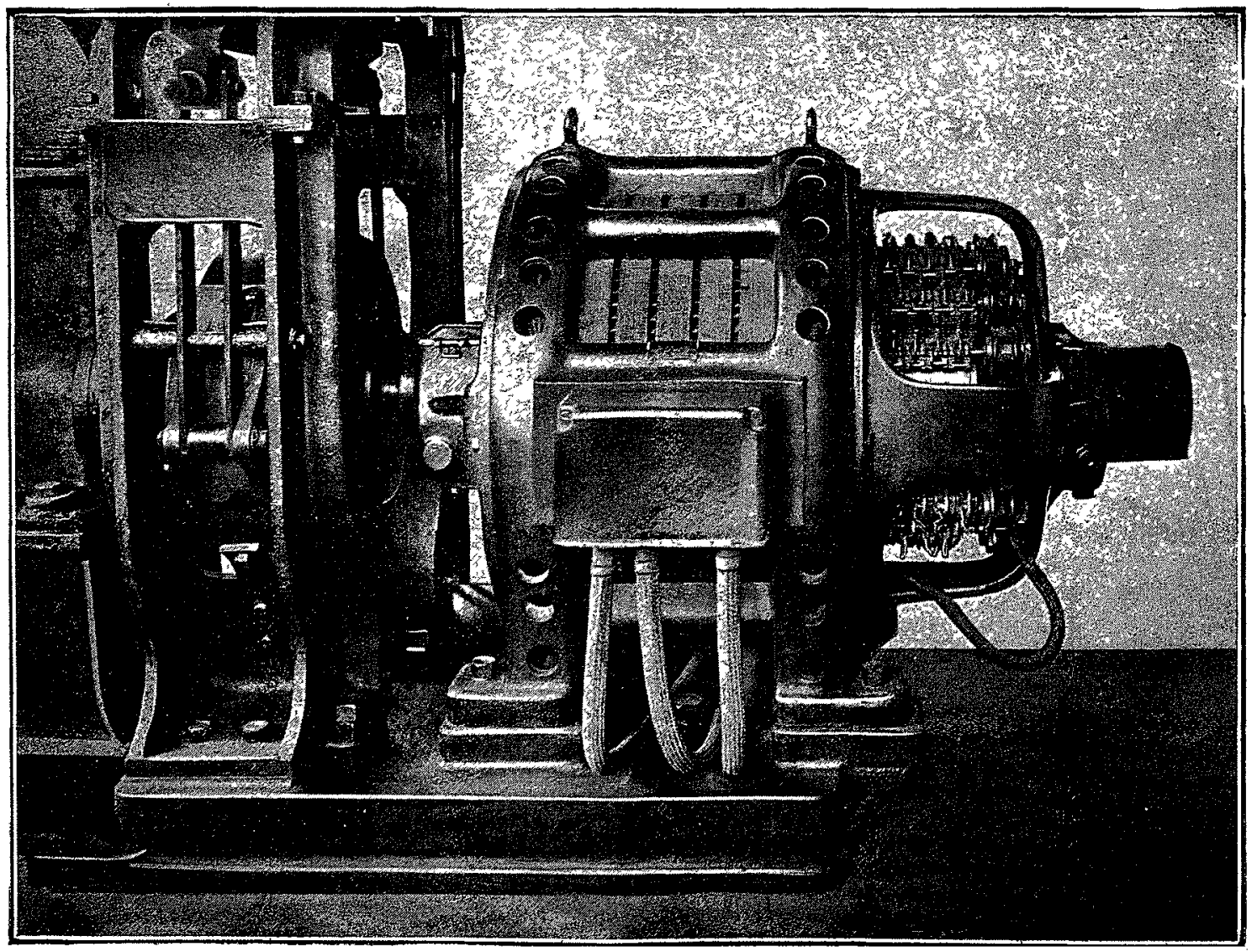

Moteur monophasé à collẹcteur série-shunt, type Latour, 55 CV., 220 V., 600 tm., actionnant un monte-charge. 
un facleur de puissance voisin de l'unilé. Le fonctionnement en auto-excilation à une fréquence vorsine de celle de la rotation n'offre pas de difficulte particulière.

7. Posstbilttés des moteurs monophasés Récapitulatıon - Les moleurs monophasés permettent de réaliser les caractérıstiques survantes :

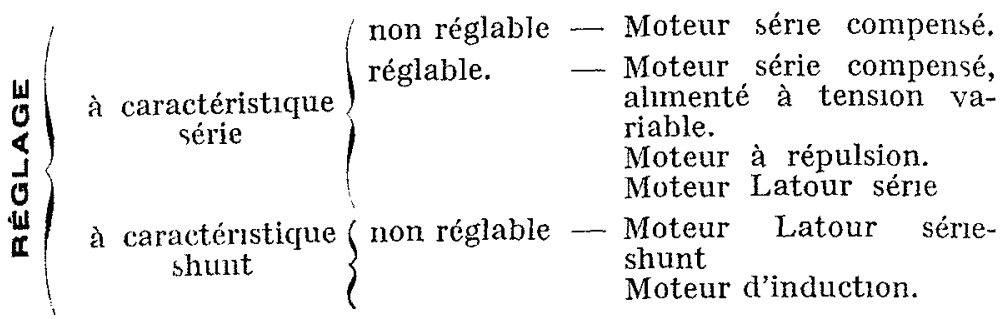

\section{Deuxième Partie}

\section{LES GÉNÉRATRICES ÉLECTRIQUES}

\author{
Chapitre Premier
}

\section{Généralités}

Le problème se pose fréquemment de convertir en énergie électrique une énergie disponble sous la forme thermique ou hydraulique. On utılise alors un moteur thermique ou une turbine, quı entraîne une génératrıce électrıque. Mais, quel genre de génératrice électıque convient-ıl de choisır ? Il importe de préciser d'abord les différentes fonctıons d'une génératrice

Une génératrice électrique fournit au crrcut récepteur connecté à ses bornes une puissance wattée $P_{w}$, qui est l'équivalent électrique de la pussance mécanıque $\mathrm{Pm}_{\mathrm{m}}$ fournie sur son arbre par le moleur d'entraînement $M$

Son rôle est également, en général, de fournur une certaine puissance déwattée, qui est absorbée par le crrcuit récepteur. Ainst, un alternateur de $10000 \mathrm{kVA} \cos \varphi 0,8$ fournit $8000 \mathrm{~kW}$ de puissance utlle et $6000 \mathrm{kVA}$ de pussance réactive au réseau. Il est plus économıque d'utilıser une seule machine pour fournir simullanément la puissance actrve et la pursance réactive, la puissance inslallée serait $14000 \mathrm{kVA}$ au heu de 10.000 , s1 l'on installat deux machmes fournissant, l'une la puissance active, l'autre la puissance réactıve

Le mot génératrice électrique s'applique même au cas d'une machıne qui fournıt au réseau auquel elle est connectée, une puissance exclusivement déwattée Dans ce cas, nous verrons qu'un moteur d'entraînement n'est plus utile. la puissance watlée nécessaire à entretenir le mouvement de rotation, peut être prise au réseau lu1-même Nous appellerons une telle machıne génératrice de puissance réactive.

Deux cas sont possibles

a) La génératrice esl connectée à un réseau à lension et fiéquence constantes Dans ce cas, la machme possède une caractéristique propre puissance-vitesse, ou caractérıtique mécanıque de la généralrice; el une caractérıłıque puissance dévattée, puissance wattée, ou caractéristique de la pussance réactive de la génératrice. Ces caractérıstıques peuvent parfors être modifıées en agıssant sur un organe de réglage. la génératrice est alors à caraclérıstıques réglables.

b) La génératrice est autonome : elle almente un réseau local directement connecté à ses bornes, à une tension et une fréquence sensibloment constantes. Ce sera le cas d'une station centrale génératrice de grande puıssance qui alimente un réseau non interconnecté avec d autres. A l'heure actuelle, dans nos pays, les réseaux de distribution s'étendent partout, et le fonctionnement autonome est exceptionnel pour les petites et moyennes puissances. Nous laisserons d'abord de côté le problème assez délicat du fonctionnement autonome, pour nous limiter au cas où ll existe un réseau.

D'autre part, le moteur d'entraînement M, peut être :

a) Un moteur à vitesse variable, fonctionnant sur sa caractérıstıque mécanique naturelle (puissance-vitesse). Il arrive que cette caractérıstique pusse se modifier; ce sera le cas d une turbıne à hauteur de chute variable, ou d'une machine dont on modifie l'admission du flude moteur.

\section{b) Un moteur muni d'un régulateur d'admission.}

Ces solutions répondent à deux besoins différents : on peut se proposer, soit de fournir au réseau un appoint de puissance constant, solt de fournir une puissance variable et réglable à volonté, de manière à suivre les varıations de la charge du réseau. Considérons successivement les deux cas.

1 er cas. - On utilise un moteur d'entraînement sans régulateur, fonctionnant sur sa caractéristıque puissance-vitesse. La génératrice travaille nécessairement à l'intersection des caractéristiques mécanıques, sa puissance est déterminée, et, par suite, la pussance déwattée est déterminée sur la caractéristıque de puissance réactive.

La génératrice fournit une puissance wattée et déwattée que le crrcull récepteur est dans l'obligation d'absorber; l'ensemble des génératrices qui almentent le réseau doit, à tout instant, sulvre les varıations de la charge wattée et déwattée du réseau.

$2^{\text {me }}$ cas - On utılise un moteur d'entraînement avec régulateur.

Le rôle du régulateur est de permettre à la génératrice de survre les variations de la charge du réseau.

II faut, d une part, un régulateur d'admission du flunde moteur, qui proportıonne le couple développé par le moteur à la charge électrique demandée par le réseau (La génératrice possédant une relation entre la charge et la vitesse définie par la caractéristıque mécanique, le régulateur d admission est, en somme, un régulateur de vitesse)

Il faut, d'autre part, un régulateur de la puissance réactive qui permette à la génératrice de débiter la puissance réactive que demande le réseau et qui est variable, en général, avec la charge de celuı-cı (par exemple, un réseau fonctionne à $\cos \varphi$ constant dans ce cas, la puissance réactive est proportionnelle à la pussance électrique fournie).

Il est à remarquer que, dans le cas où la génératrice est connectéc à un réseau à tension et fréquence constantes, de puissance élevée par rapport à celle de la génératrice, le fonctionnement sans régulateur est le seul à consıdérer.

En solnme, une génératrice électrıque est utilisée, en général, pour deux catégorles d'applucations :

10 On se propose d'installer une génératrice dans le but d'alimenter des récepteurs électriques : moteurs, lampes, appareils de chauffage. La génératrice dort alors fonctionner autonome et suivre les varuations de la charge La génératrice sera établie pour' s'adapter au mieux à l'alımentation des récepteurs.

En partıculier, la génératrice doıt être capable des mêmes surcharges que les récepteurs.

20 Il exısle, d'une part, un réseau d'énergıe électrique, et, d'autre part, unc source naturelle d'énergıe mécanique, hydraulıque, thermıque. On désire convertir cette énergie naturelle sous la forme électrique, pour fournir un appoint au réseau. La génératrice ne fonctionne alors jamais en surcharge.

C'est le second problème que nous avons seul en vue dans le chapitre suivant, avec cette précision supplémentaire que la puissance du réseau (wattée et déwattée) est grande par rapport à celle de la génératrice. 
Chapitre II

\section{Les génératrices de réseau}

Nous considérerons d'abord, à titre d'introduction :

$1^{\circ}$ en courant conlmu, la machme shunt, qui est la plus smple des génératrices asynchrones.

$2^{\circ}$ en courants alternatifs, l'altennaleur synchrone, qui a été longtemps la seule machme employée.

Nous en aruverons alors à l'objet principal de cette étude, la génératrice d'unduction polyphasée

\section{I. - Géneratrice astinchrone shun't a COURANT CONTINU}

Cette machune est toute désignée pour servir d'introduction à notre étude, car les relations sont très simples, du fait que seule la puissance wattée intervient.

Supposons donc qu'il existe un réscau d'énergı électrique à courant continu et tension conslante.

A) Caractértstique mécanıque de la génératrıce ( $1 \mathfrak{\text { g. 82) }}$ - - La caractéristique de vitesse en fonction du couple se calcule aisé-

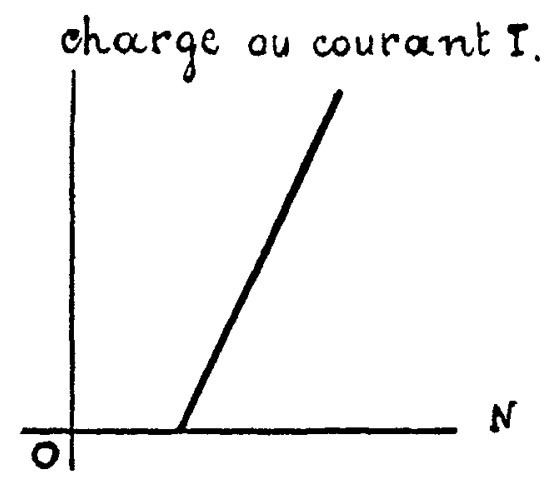

FIG. 82. - Génératrice shunt, caractéristique mécanique.

ment, sı on peut négliger la réaction de l'induit, car la machine fonctionne alors à flux constant

A vide, la force électro motrice $E$ est égale à $U$; la vitesse prend alors la valeur $\mathrm{N}_{\mathrm{v}}$, et l'on a :

$$
\mathrm{U}=\mathrm{E}=\mathrm{K} \mathrm{N}_{\mathrm{v}} \Phi
$$

$K$ désignant une constante.

En charge, on aura

$$
\begin{gathered}
E-U=R I \\
E=K N \Phi
\end{gathered}
$$

Par suite

$$
\frac{\mathrm{N}-\mathrm{N}_{\mathrm{v}}}{\mathrm{N}_{\mathrm{v}}}=\frac{\mathrm{RI}}{\mathrm{U}}
$$

La vitesse augmente quand la charge augmente, l'accrorsement de vitesse entre vide et charge est proportionnel à la charge.

Si l'on intercale une résıstance en série avec l'induit, on peut obtenir des inclinaisons croissantes de la caractéristıque $S_{1}$ on intercale un rhéostat d'excitation, le point $\mathrm{N}_{\mathrm{v}}$ peut être déplacé : la caractéristıque se déplace parallèlement La génératrice asynchrone est annsı une génératrice à caractéristique réglable (fig. 83).

в) Moteur d'entraînement sans régu'ateur. - Si la machine est entra^née par un moteur qui suit sa caracléristique naturelle $\mathrm{C}_{2}$ du couple en fonction de la vilesse, le point de fonctionnement se fıxe à l'intersectıon des caractéristıques, comme on l'a déjà expliqué
En modrianl l'exciation, on peul oblenir tous les pomts d'intersection de $\mathrm{C}_{2}$, avec les caractéristiques successives du

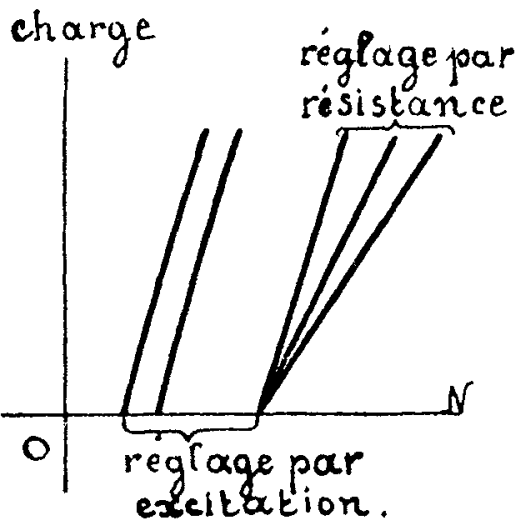

Fic. 83. - Génératrice shunt à caractéristrque réglable

couple de la généralrice : le réglage de la charge est annsi possibl, dans une certame mesure, par l'excitation (fig. 84)

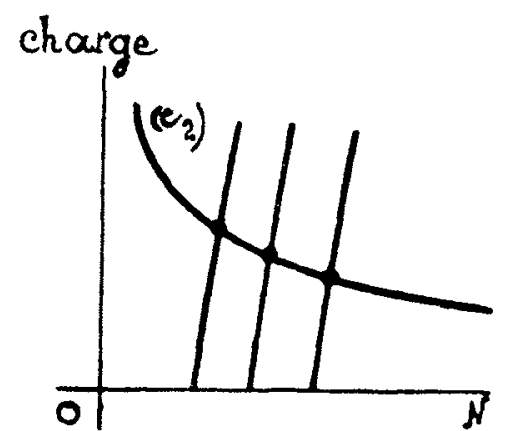

FIG. 84. - Génératrice shunt, Iróglage de la charge par l'excitatioll,

Un cas d'application intéressant est le suivant : on utilist une génératrice comme machine de charge pour convertır en énergie électrique, l'énergie d'un moteur qui sut sa caractéris tıque naturelle, mais dont la caractéristıque naturelle du couph en fonction de la vitesse, peut être modifiée (cas d'une turbine à hauteur de chute variable)

Les points de fonctionnement sont alors à l'intersection du réseau des courbes couple-vitesse du moteur, avec le réseall des courbes correspondantes de la génératrice (fig. 85).

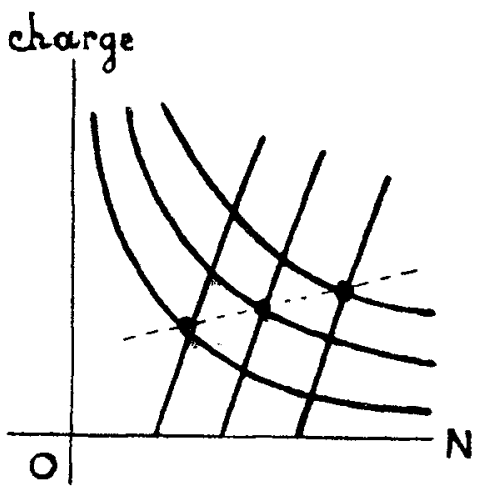

Fig. 85. - Génératrice shunt, intersectıon des caractérıstıques.

En général, pour la bonne utilisation du moteur, al convienl de fonctionner en un point partıculier de sa caractérıstique: le réglage de l'excitation permel d'amener la caracléristique de la génératrice à passer par le point de la caractérislıque dt couple moteur où l'on désire fonctionner. Le heu de ces points de fonclionnement est la courbe pointillée, fig. 85.

c) Couplage sur le réseau. - Comme il est bien connu, il faul amener la machine, excrtée séparément, à une tension égale opposée à celle du réseau, avant de la coupler. 


\section{1. - Machine SYNCImone A CoURANTS alternatifs}

Dans le cas d'un réseau polyphasé, la génératrice type est l'allernaleur synchrone, dont nous allons rappeler les propriétés.

A) Caractéristique mécantque de la génératrice. - La génératrice ne possède pas de caractérıstıque mécanique, car la vitesse est une constante. la machine synchrone ne peut fonctionner en parallèle que si elle lourne à une vitesse rigoureusement détermince, égale à la vitcsse de synchronisme $\mathrm{N}_{\mathrm{s}}$ déterminée par sa polan lé cl la fréquence du réseau. Le couple dépend, non de la vilesse, mâs de l'écart angulaire (angle de l'axe d'un pôle avec l'axe du champ d'indul).

B) Caractértstlque de putssance réactive. - Cette caractérılique à excitation conslante cst donnée par le diagramme circulaire clashique Quand on augmente l'excilation, le diamètre de la cuconférence augmente (fig 86).

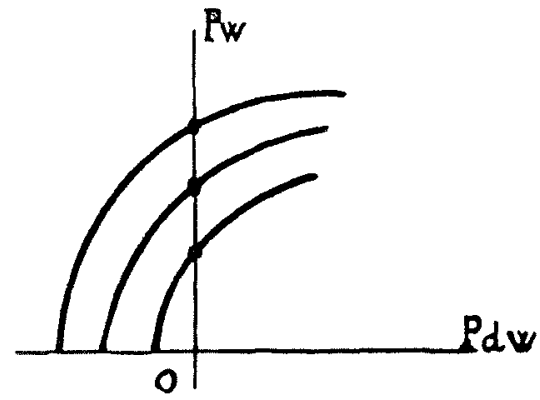

Fis 86 - Génératice synchrone, puissance réactive.

c) Moleut d'entraînement sans régulateur. - Le point de fonctionnement (lig 87) est celui qui correspond à $\mathrm{N}=\mathrm{N}_{\mathrm{s}}$.

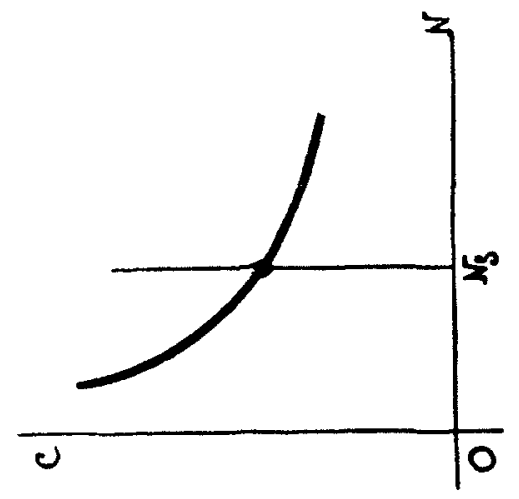

FIG. 87. - Génératrice synchrone, point de fonctionnement.

Le moteur d'entraînement est donc astreınt à fonctionner à une vitesse fixe, tant que la fréquence reste invariable.

$\mathrm{Si}$ le moteur d'entraînement possède une caractéristique peu Inclinée (fig. 88), c'est-à-dure une vitesse qui baisse peu

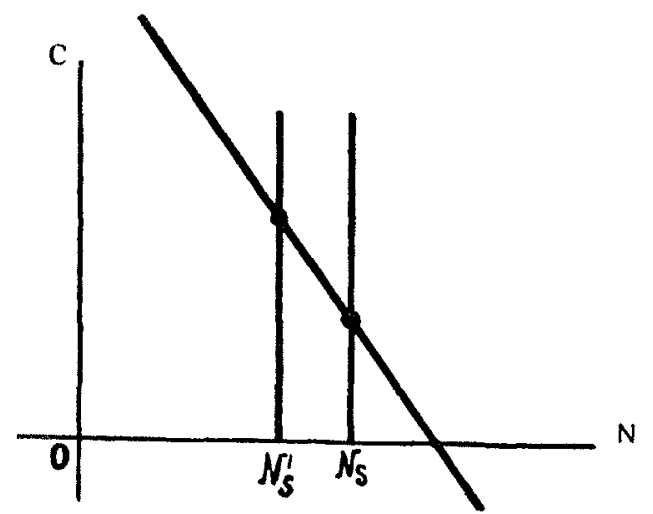

Fig 88. - Géné ratrice synchrone, variation de la charge avec la fréquence.

quand la charge augmente, alors, le point d'intersection avec la verticale $\mathrm{N}=\mathrm{N}_{\mathrm{s}}$ est sujet à varier beaucoup pour une légère variatıon de la fréquence du réseau . le fonctionnement est peu stable. Si le moteur d'entraînement est un moteur synchrone, il est impossible de charger la génératrice, car on ne peut concevoir qu'un couple se transmette d'une machine à l'autre.

Le moteur d'entraînement doit constamment tendre à augmenter la vitesse de la génératrice : bien qu'll fonctionne à vitesse constante, on ne peut pas prendre pour assurer ce service, un moteur dont la vitesse est constante. C'est là une circonstance d'apparence paradoxale.

En cas de manque de tension sur le réseau, la machine perd sa vitesse de synchronisme, si on rétablit alors la tension, ll se produit un véritable court-circuit. La machine ne peut reprendre d'elle-même le synchronisme, puisqu'elle ne possède aucun couple en dehors du synchronisme.

D) Fonctionnement en génératrice de déwatté. — On vout sur la caractéristique de pussance réactive que si la puissance utile diminue progressivement, la machine débite une puissance magnétisante qui croît constamment. Si l'on supprime le moteur d'entraînement, la machine fonctionne sur la partie du diagramme symétrique correspondant à la marche en moteur, la puissance utile prise au réseau correspondant seulement aux pertes. Le pount de fonctionnement est très volsin de l'axe. On dit que la machine fonctionne en compensateur synchrone.

Dans le cas où la source d'énergie qui alimente le moteur d'entraînement vient à s'arrêter, la machine électrique peut être utilisée comme génératrice de puissance réactive, et le réseau Iui fournit la puissance wattée très réduite nécessaire pour entretenir le mouvement de rotation.

E) Couplage sur le réseau. - Pour mettre une machine synchrone en service, 1 faut d'abord l'amener, à vide, à une tension égale à celle du réseau, puis établir la concordance de phase entre sa tension et celle du réseau : une manœuvre de couplage est nécessaure.

\section{III. - GÉNÉRATrice D'INDUCTION SIMPLE}

On a été conduit à substituer souvent la génératrice d'induction à la génératrice synchrone, pour différentes raisons, qui résultent des propriétés que nous allons indiquer.

A) Caractértstique mécanique de la génératrice (fig. 89). — Cette

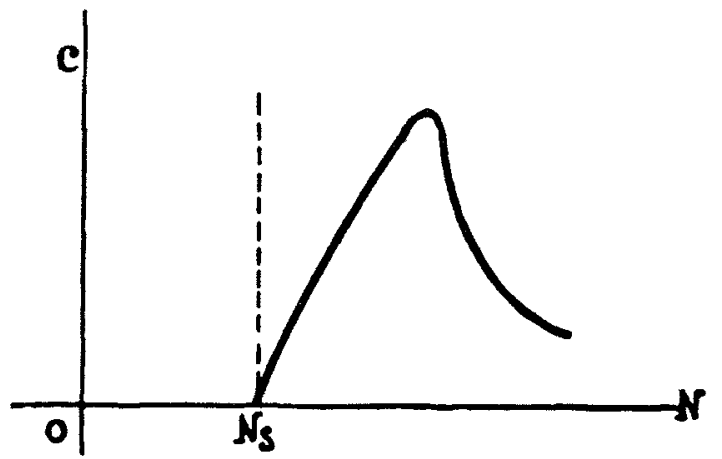

Fig. 89. - Couple de la génératrice asynchrone.

caractéristique est donnée par la branche en génératrice du diagramme circulaire classique du moteur d'induction. Le seul moyen d'augmenter la charge est d'augmenter la vitesse, comme pour la machine shunt à courant continu.

La génératrice asynchrone n'est donc pas une machine synchronisée : elle ne peut se charger qu'en raison de son écart de vitesse par rapport au synchronisme, et la fréquence à laquelle elle fonctionne est déterminée, non par sa vitesse de rotation, mais par la fréquence du réseau.

в) Caractéristique de puissance réactive (fig. 90). - La caractéristique de la puissance réactive est représentée par le diagramme circulaire. A vide, la machine prend au réseau la puissance 
d'excitation du circuit magnétıque principal, en charge, cette puissance est augmentée des puissances déwattées de fuites.

c) Moteur d'entraînement sans régulateur. - La vitesse se fixe automatiquement au point d'intersection des caractéristiques.

En cas de manque de tension sur le réseau, le moteur d'entraînement tend à prendre sa vitesse d'emballement, car, toute

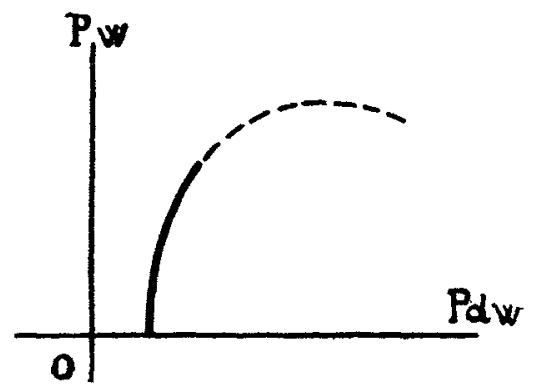

FIG. 90. - Génératrice d'inductıon, puissance réactıve.

excitation disparaissant, la machine asynchrone ne possède plus de couple. Quand la tension est rétablie, l'ensemble "ne peut revenir de lui-mème au point de fonctionnement normal que si le couple résistant de la génératrice est plus grand que le couple du moteur d'entraînement (fig. 91).

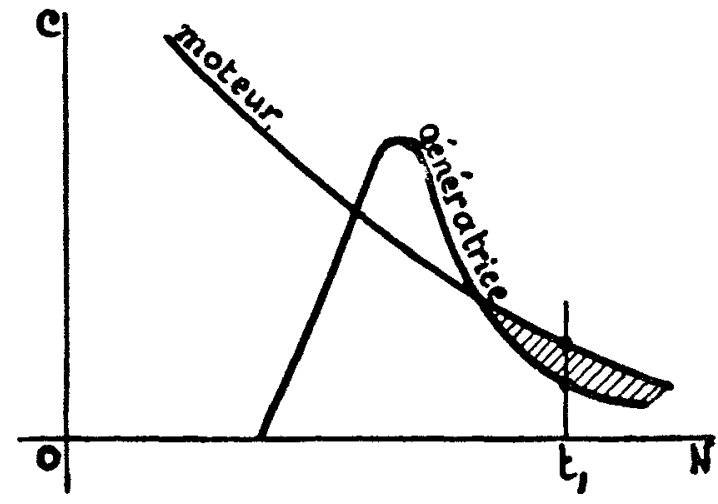

FIG. 91. - Génératrice asynchrone, cas d'emballement du moteur.

Au contraire, si la tension se rétablit à un moment tel que $t_{1}$, où la vitesse est telle que la courbe du couple moteur solt audessus de celle du couple résistant, la vitesse continue à augmenter. Le courant augmente de plus en plus, et la machine prend un courant voisin de son courant de court-circuit en attelgnant sa vitesse d'emballement.

D) Mise en serv،ce sur le réseau. - La génératrıce asynchrone est une machine dont la mise en servace sur le réseau est très simple, car aucun couplage n'est nécessaire . il suffit de démarrer le moteur d'induction sur rhéostat en moteur à vide, et, quand le synchronisme est atteint, d'ouvrir l'admission du moteur d'entraînement pour lu farre prendre la charge

Si l'on a ouvert l'admission du moteur d'entraînement dès le début de la mise en route, alors le couple sur l'arbre de la machine asynchrone est égal au couple moteur fourn par la machine motrice, augmenté du couple du moteur d'induction. La somme de ces deux couples provoque une accélération très rapıde. On diminue progressivement la résıstance rotorıque jusqu'au court-circuit, mais, si l'on ne va pas assez vite, et que le moteur a déjà atteint une vitesse hypersynchrone quand le rhéostat est encore partiellement en circuit, la vilesse peut atteindre une valeur très supérıure à la vitesse normale, du fait que, avec résıstance rotorıque, comme nous l'avons vu (fig 40), l'accroissement de vitesse entre vide et charge peut atteundre une valeur très élevée dans le fonctionnement en génératrice d'induction.
On peut également, à l'aide du moleur, entraîner la machin d'induction, et fermer alors son stator sur le réseau, sans mise en phase à une vitesse légèrement inférieure au synchronısme. La machine prend alors sa charge.

Il est nécessaire que le rotor soit entraîné à une vilesse voisine du synchronisme pour éviter un à-coup de courant quand lo stator est fermé sur le réseau : à-coup qui est déterminé par l'or donnée de la courbe couple-vitesse du moteur d'induction, pour la vitesse qu'll possède à l'mstant de la fermeture.

Si le rotor tourne à une vitesse sensiblement inférieure an synchronisme, on évitera tout à-coup, en intercalant une résis tance rotorique convenable, qui incline la caractéristique couple vitesse.

\section{IV. - GÉNÉRATRICE D'INDUCTION AVEG EXGITATRIGE POLYPHASÉE}

Avant de comparer la génératrıce synchrone avec la généı atril d'inductıon, nous indıquerons une amélıoration capitale apporter à la machine d'induction simple.

Cette amélioration consıste en l'adjonction d'une excitatric à collecteur polyphasée, qui est aussi indispensable à la machine d'induction que l'excitatrice à courant continu l'est à l'alter nateur synchrone.

L'excitatrice à collecteur est, en principe, constituée par une machine comportant bagues et collecteur, reliée, d'une par par son collecteur polyphasé aux bagues du moleur d'induction et, d'autre part, par ses bagues au réseau.

Il existe deux formes de réalisation :

$1^{\circ}$ L'excitatrice est montée en bout d'arbre du moteur prin cipal (fig. 58).

$2^{\circ}$ L'excitatrice est combinée avec le moleur principal, sous forme d'une machine unique, dite : machine d'mduction aux compensée (fig. 42).

Ces deux dispositions sont équivalentes.

A) Caractéristique mécanıque de la génératrice. - La caract ristique vitesse-charge dans le fonctionnement en génératrie est une caractérıstıque shunt de même allure que celle de machine d'induction simple.

B) Caractéristıque de la pulssance réactive (fig. 92). -

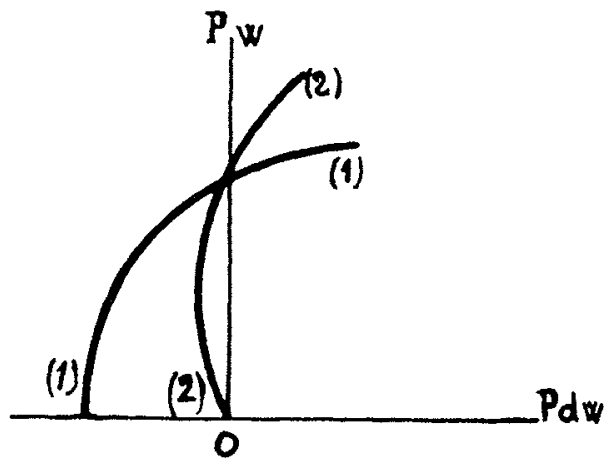

FIG. 92. - Génératrice d'induction, puissance réactıve.

caractéristıque des $\mathrm{kVA}$ réactıfs en fonctıon de la puissant électrique débitée, est toul à fait comparable à celle obtelue avec une génératrice synchrone à excilation conslante.

Mars, avec la présente machine, ll est encore possible d'obtelue au lieu de l'allure (1), une courbe d'allure (2) telle que la pus sance magnétısante, au lieu de décrô̂lre constamment qua la charge augmente, reste sensıblement nulle entre vide et plein charge.

c) Moteur d'entraînement sans régulateur. - La mac entraînée par un moteur sans régulateur peut être utilisée lieu et place d'un alternateur synchrone à excitation constant dans tous les cas d'emploi de cette machine. On peut égaleme 
J'utilıser à puissance nulle, pour fournir exclusivement de la puissance réactive au réseau : exactement comme un compensateur synchrone.

L'ensemble machine synchrone-excitatrice à courant contınu est alors remplacé par l'ensemble machine asynchrone - excltatrice polyphasée. On obtient alors un compensateur asynchrone

Il est possible de régler la puissance magnétısante débitée par la génératrice asynchrone, pour une puissance wattée déterminée, simplement en décalant les balais. Mais la vitesse se fixe alors à une valeur légèrement différente.

De même, dans le cas d'un compensateur asynchrone : si l'on règle la puissance magnétisante débitée, la vitesse da la machine varie légèremcnt, ce qui est sans importance.

Comparaison des génératrices synchrone et d'induction compensée - Comme nous venons de le vour, les génératrices asynchrones avec excitatrice de compensation polyphasée, et synchrones avec excrtalrice à courant continu, ont les mêmes proprétés générales, qu'elles soient utilisées comme génératrices de puissance ou comme génératrices de déwatté.

La question se pose donc de comparer les deux solutions.

Remarquons d'abord qu'une fluctuation de fréquence entraîne dans une machine synchrone un pompage et des oscillations pendulaires de la roue polaire, auxquelles on peut, dans une certaine mésure, remédier par l'emploi d'enroulements amortisseurs de très faible résistance. Dans le cas de la machine asynchrone, il y a simplement une yariation de glissement, et la machme se réadapte aux nouvelles conditions sans oscillations.

Mise en court-circuit. - La mise en court-circuit est un phénomène accidentel qui est, en général, très dangereux pour une génératrice. On comprend donc que les exploitants attachent une importance partıculière à la façon dont la machine se comporte en pareil cas.

Il convient de distinguer la période initiale du court-circuit, et le régıme permanent qui s'établit ensuite.

$1^{0}$ Période initıale. - Le courant atteint en quelques milhèmes de seconde, une valeur considérable. cette valeur ne dépend que de la réactance de fuites combinée du stator et du rotor. Avec un rotor qui possède un enroulement amortisseur peu résistant, ce qui est toujours le cas de la machine synchrone, la réactance de fuites combinées se réduit à celle du seul stator.

Au contraire, avec rotor non amorti (ce qui est le cas de la machine asynchrone), la réactance de fuites du rotor intervient; le courant de court-circuit instantané sera donc moindre.

Comme les efforts sur les bobinages varient avec le carré du courant, on conçoit tout l'intérêt de cette réductıon du courant initial.

$2^{\circ}$ Régime. - Le courant se stabilıse à une valeur déterminée, si l'excitation est maintenue; au contraure, il s'annule évidemment si on fait disparaître l'excitation.

Or, avec la machine synchrone, le circuit d'excitation n'est pas intéressé par le court-circuit; l'excitation persiste donc à sa pleine valeur pendant le court-circuit, et le courant de courtcircuit se fixe à 2 à 3 fois le courant normal. Il faut prévorr un rupteur d'excitation pour l'annuler.

Le cas est comparable à la mise en court-circuit d'une machine à courant continu à excitation séparée.

Au contraire, avec la machine asynchrone, la mise en courtcircuit a pour conséquence de faire automatiquement disparaître l'excitation : le courant permanent est donc nul, et cela sans aucune manouvre.

Le cas est comparable à la mise en court-circuit d'une machine shunt.

On conçoit l'intérêt capital que présente la disparition de l'excitation, qui aggraverait très rapidement les effets nuisibles en alimentant le court-circuit.

Mise en service. - La mise en service de la machine asynchrone se fait par simple fermeture d'un interrupteur en un ou deux temps on évite le couplage nécessaire à la génératrıce synchrone, et, par sute, un appareillage automatique peut être facilement envisagé.

Considérons maintenant plus particulièrement le fonctionnement en génératrıce de puissance et génératrice de déwatté.

Génératrice de puissance. - Variations de charge et oscullations de vitesse de la machine motrice. - Une variation brusque de la charge ne peut entraîner de pompages, avec la génératrice asynchrone, pour la raıson déjà indıquée en cas de fluctuatıon de fréquence.

Une fluctuation périodique de vitesse de la machine motrice, comme 1 l en exıste dans le cas des moteurs à gaz à couple périodiquement variable dans le tour, demande certaines conditions de $\mathrm{PD}^{\mathbf{2}}$ avec la machine synchrone pour éviter le décrochage.

$\mathrm{Au}$ contraire, avec la génératrice asynchrone, le coefficient d'irrégularité est sans importance.

Génératrice de détuatté. - A) Baisse momentanée de tension. Si la tension baisse, le couple de la machıne synchrone ou asynchrone diminue, et il peut s'ensuivre un décrochage. Comme le couple maximum est beaucoup plus élevé pour la machine-asynchrone avec excltatrice polyphasée, ll faudra une plus grande baisse de tension pour la décrocher.

En outre, la machine asynchrone doit atteindre un certain glissement avant de décrocher; il faut un certain temps pour atteindre ce glıssement, ce qui laissera à la tension le loisir de se rétablur pendant le temps nécessaire aux masses tournantes pour libérer leur énergie cinétıque.

B) Appel de courant au démarrage. - L'appel de courant au démarrage est très rédunt avec la machine asynchrone. Il est de l'ordre du $1 / 3$ du courant normal ou moindre avec démarrage étoile-triangle. Une machine synchrone demande, au contraire, au démarrage sous tension réduite, quı est le mode de démarrage le plus répandu, un courant vorsin du courant normal.

c) Débit maximum de puissance réactive $A V$ et $A R$. - Dans le cas où on utılise une génératrıce de puissance réactive pour régler la tension d'un réseau, ll convient de pouvorr utıliser la machine pour débiter des $\mathrm{kVA}$ avant dans le fonctionnement en charge, afin de rédure la chute de tension due au courant déwatté arrière crrculant dans la ligne, et d'absorber des kVA avant dans le fonctionnement à vide afın de réduire l'élévation de tension due au courant de charge de la ligne. La courbe en $\mathrm{V}$ de la machine synchrone montre que l'on ne peut aller aussi loin en kVA arrière qu'en kVA avant, par suite des risques de décrochage à excitation très réduite. Avec la machne asynchrone, on peut obtenir les mêmes kVA dans les deux cas.

D) Variation de tension du réseau. - Pour les deux machines, la courbe des $\mathrm{kVA}$ débités à excitation constante (ou calage constant) est une fonction de la tension aux bornes qui présente la même allure : quand la tension augmente, les kVA augmentent, passent par un maximum, puis diminuent.

Toutefois, pour la machine asynchrone, le maximum est plus aplati, car il se produit une compensation, en cas d'augmentation de la tension du réseau, entre l'augmentation du courant magnétisant et l'augmentation de la tension d'excitation.

\section{V. - GÉnÉratrice D'INDUCTION AVEC EXCITATRICE DE GLISSEMENT}

La génératrice d'induction qui vient d'être décrite est une machine à vitesse constante. Mais l'emploi d'une excitatrice, 
dite de glissement, permet d'obtenir un fonctionnement à vitesse réglable.

L'excitatrice de glıssement est unc machine analogue à celle quı a été décrite pour la compensation, mais, au lieu de prendre la tension aux bagues de l'excitatrice sur le réseau, on la prend sur le secondaire d'un transformateur à rapport réglable, dont le primaire est alimenté par le réseau. Le réglage du rapport de transformatıon permet de régler la vitesse à vıde.

La machine d'induction avec excitatrice de glıssement, présente les différences survantes, par rapport à la machıne d'ınduction simple :

La caractéristıque du couple (ou de la puissance) en fonction de la vitesse, peut être déplacée parallèlement à elle-même, dans certaines limites (fig. 93).

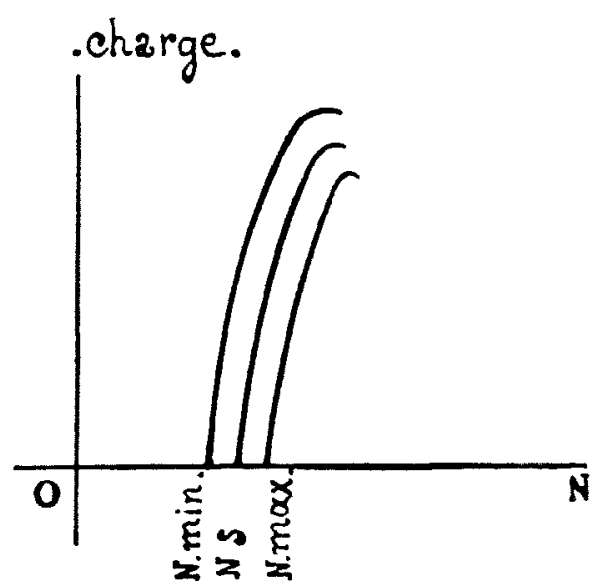

Fig. 93. - Génératrice asynchrone à vitesse variable, caractéristıques Vitesse-charge.

A chaque caractéristique puissance-vitesse correspond une caractéristique des $\mathrm{kVA}$ échangés en fonction des $\mathrm{kW}$ débités sur le réseau (fig. 94).

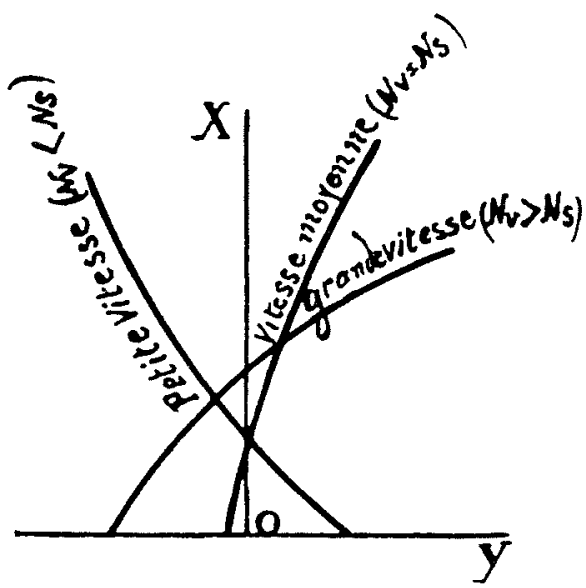

Fıg. 94. - Génératrice asynchrone à vitesse variable, puissance réactive.

Le fonctionnement est défini pour ces deux faisceaux de caractéristiques.

Applications. - Ces génératrices trouvent leur emploi dans les cas suivants :

$1^{\circ}$ On veut convertir en énergie électrique, l'énergie mécanique disponible sur un arbre à vitesse variable, cette énergie étant bien déterminée pour chaque vitesse. C'est là un problème que la machine shunt permettait de résoudre, comme nous l'avons vu dans le cas du courant continu.
La génératrice asynchrone permet d'obtenir une lamille courbes couple-vitesse sur chacune desquelles on uhlise seul point, correspondant aux conditions optima de la turb motrice, que l'on appellera point normal (fig. 95)

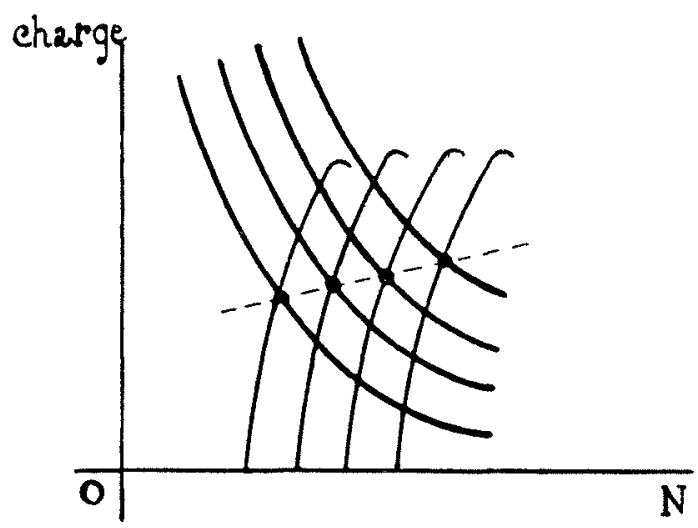

FIG. 95. - Génératrice asynchrone à vitesse variable, intersection des caractérıstiques.

Il convient, en général, d'établır la génératrice de lelle sor que la caractéristique pussance -wattée, pussance déwatt correspondant à chaque vitesse, passe par $\cos \phi=1$ pour point normal.

$2^{\circ}$ On veut alımenter deux réseaux de fréquences différent à l'aide de deux génératrıces entraînées par le même moteur, régler à volonté la puissance débitée dans chacun des résea: On utilise dans ce cas une génératrice synchrone tournant à vitesse $\mathrm{N}_{\mathbf{S}}$ constante, et une génératrice asynchrone avec vites à vide réglable par excitatrıce de glıssement.

Désıgnons par $\mathrm{P}_{1}$ la pleıne charge de la génératrıce asynclire et proposons-nous d'obtenir la pleine charge de celte génít trice à la vitesse $\mathrm{N}_{\mathrm{S}}$ de rotation, maintenue constante. Il suff d'utiliser la caractéristique (C') comme représenté sur la figure

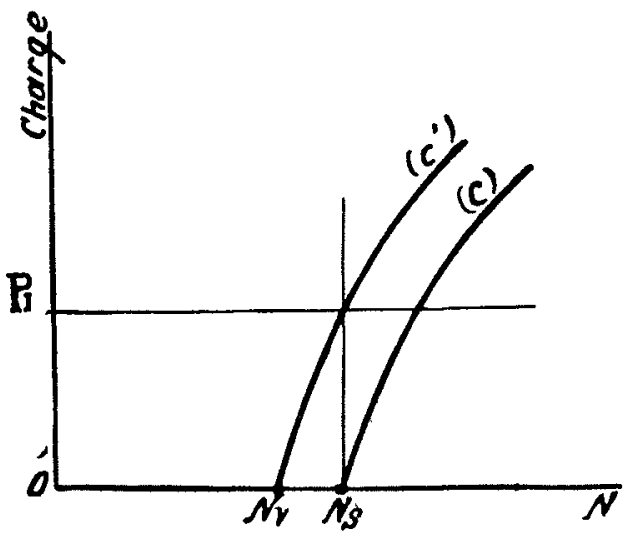

FIG. 96.

En modıfiant la vitesse à vide de la valeur $\mathrm{N}_{\mathrm{s}}$ à la valeur ce qui est possible par modificalion du rapport du transfort teur alimentant les bagues de l'exchtatrice, on fera passer toll la charge sur la génératrice asynchrone.

Conclusion. - Il résulte des considérations qui précèdent, $\sharp$ pour convertir une énergie naturelle en appoint d'énergie trique transmis à un réseau polyphasé, la génératrıce synchro constitue la solution ancienne et démodée, landis que la gént trice asynchrone sous sa forme moderne, c'est-à-dire solls forme compensée séparément ou auto-compensće, constitue solution la plus rationnelle et la plus souple, et permet d'abor les problèmes les plus variés.

(Extrait de Jeumont, avril-juin 1928).
(A suivre.) 